\title{
Deoxycholic Acid and Lithocholic Acid Alleviate Liver Injury and Inflammation in Mice with Klebsiella pneumoniae-Induced Liver Abscess and Bacteremia
}

This article was published in the following Dove Press journal:

Journal of Inflammation Research

\author{
Yahong Zheng' \\ Chengcheng Yue' \\ Hui Zhang' \\ Haoran Chen' \\ Yanyan Liu' \\ Jiabin $\mathrm{Li}^{\mathrm{I}-4}$
}

'Department of Infectious Diseases, The First Affiliated Hospital of Anhui Medical University, Hefei, People's Republic of

China; ${ }^{2}$ Department of Infectious

Diseases, The Chaohu Affiliated Hospital of Anhui Medical University, Hefei, People's Republic of China; ${ }^{3}$ Anhui Center for Surveillance of Bacterial Resistance, Hefei, People's Republic of China; ${ }^{4}$ Institute of Bacterial Resistance, Anhui Medical University, Hefei, People's Republic of China
Correspondence: Jiabin Li

Department of Infectious Diseases, The First Affiliated Hospital of Anhui Medical University, Jixi Road No. 218, Hefei, 230022, People's Republic of China

Tel +86-55I-629227I3

Fax +86-55I-6292228I

Email lijiabin@ahmu.edu.cn
Purpose: Klebsiella pneumoniae-induced liver abscess and baiacterem is a serious infectious disease with high mortality. Secondary bile acids (SBAs) are produced by intestinal flora through the metabolism of primary bile acids and play a role in promoting or inhibiting inflammation in some diseases. However, the immunomodulatory role of SBAs in bacterial infections of the liver remains unclear. This study aimed to investigate the anti-inflammatory and liver-protective effects of SBAs in $K$. pneumoniae-infected mice.

Methods: The absolute concentrations of deoxycholic acid (DCA) and lithocholic acid (LCA) in feces and serum were analyzed, and intestinal flora alterations between $K$. pneumoniae-infected and healthy control mice were examined. The effect of SBAs was investigated by analyzing the survival, tissue bacterial load, histopathology, and inflammatory factor levels in SBA-treated mice. The expression of crucial proteins implicated in the NF- $\kappa \mathrm{B}$ pathway, as well as the G-protein-coupled bile acid receptor TGR5, was detected.

Results: The content of SBAs in feces and serum of the $K$. pneumoniae-infected group was significantly reduced, and significant changes in the composition of the intestinal flora were detected. The intestinal flora are directly related to the synthesis of SBAs. Ruminococcaceae levels in K. pneumoniae-infected mice were significantly lower than in healthy control mice. Oral administration of SBAs improved the survival and liver pathology of K. pneumoniaeinfected mice, and reduced the bacterial load and the level of inflammatory factors. SBAs down-regulated the expression of key proteins in the NF- $\mathrm{KB}$ inflammatory signaling pathway, including the phosphorylation of $\mathrm{I} \kappa \mathrm{B} \alpha$ and NF- $\kappa \mathrm{B}$ p50 and the nuclear translocation of NF$\kappa \mathrm{B}$ p65. The protective effect of SBAs may be dependent on high TGR5 expression.

Conclusion: SBAs downregulate the NF- $\kappa \mathrm{B}$ inflammatory signaling pathway through TGR5, protecting the liver and inhibiting inflammation in $K$. pneumoniae-induced liver abscess and bacteremia.

Keywords: Klebsiella pneumoniae liver abscess, deoxycholic acid, lithocholic acid, inflammation, nuclear factor- $\kappa \mathrm{B}$

\section{Introduction}

Klebsiella pneumoniae, a member of the Enterobacteriaceae family, is a gram-negative, encapsulated, rod-shaped bacterium that is the leading cause of bacterial liver abscesses and bacteremia. ${ }^{1-3}$ Studies have shown that hypervirulent $K$. pneumoniae (hvKp) account for $80 \%$ of all liver-abscess-causing strains. ${ }^{4}$ Unlike the classic $K$. pneumoniae 
(cKp) that infects elderly patients with immunodeficiency, hvKp can severe invasive infections such as endophthalmitis, necrotizing fasciitis, and meningitis, ${ }^{5}$ in healthy young people without potential comorbidities. ${ }^{6}$ In the last two decades, a distinct invasive syndrome, namely $K$. pneumoniae liver abscess (KLA), has been increasingly reported in Asia and is emerging as a global disease. ${ }^{7-9}$ Therefore, it is critical to find new and effective treatment methods for KLA and bacteremia.

Intestinal flora is the subject of intense research interest in the field of life sciences and health. In the healthy host, numerous microorganisms form a microbial ecosystem that coevolves to establish a mutualistic relationship with the host and participates in host metabolism, tissue development, and immune response. ${ }^{10}$ Multiple lines of evidence have demonstrated that dysbiosis of the intestinal flora underlies the pathogenesis of, or promotes, liver disease, including alcoholic fatty liver, ${ }^{11}$ non-alcoholic fatty liver, ${ }^{12}$ and cirrhosis. ${ }^{13,14}$ Therefore, an in-depth understanding of the changes in intestinal flora associated with liver abscesses may be beneficial for the prevention, diagnosis, and treatment of KLA and bacteremia.

Microbes are known to produce bioactive compounds that affect intestinal inflammation. ${ }^{15}$ Secondary bile acids (SBAs), whose concentration is the highest among intestinal metabolites, are originally derived from primary bile acids (PBAs) in a process reliant on microbial biosynthesis. ${ }^{16,17}$ In fact, only a few known intestinal flora (all from the Lachnospiraceae and Ruminococcaceae families) perform the subsequent 7adehydroxylation step, respectively generating deoxycholic acid (DCA) and lithocholic acid (LCA) (the two most common SBAs) to participate in immunity and the inflammatory response. ${ }^{18-20}$ A large number of previous studies have focused on the cytotoxic effects of bile acids. For example, chronic exposure to high concentrations of hydrophobic bile acids can stimulate carcinogenesis in the digestive system. ${ }^{21}$ Studies have also found that bile acids, such as LCA and DCA, are the important risk factor for gastrointestinal cancer. ${ }^{22}$ Interestingly, new evidence has revealed the beneficial role of BAs in some diseases. For instance, in the dextran sodium sulfate (DSS) murine colitis model, LCA plays a barrierprotective role in preventing the release of pro-inflammatory factors from colonic epithelial cells. ${ }^{23}$ In addition, in chikungunya virus infections, researchers have also found that DCA supplementation enhances the activity of the plasmacytoid dendritic cell-type I interferon signal axis to regulate the host's antiviral immunity and the amount of circulating virus, thus limiting the spread of the new virus. ${ }^{24}$ Bacterial liver abscess is a common infectious disease of the liver, and the regulatory effect of SBAs on its pathogenesis is still unclear. Based on previous studies, it is reasonable to assume that bile acid stimulation has a protective effect on KLA, and the main purpose of the study was to verify whether bile acid stimulation has this effect. Moreover, previous studies have found that LCA exerts its effects through the TGR5, a suppressor of NF-kB-mediated inflammation. ${ }^{25}$ It is therefore reasonable to assume that TGR5 mediates the effect of SBAs on KLA. So we also tried to explore whether the role of bile acids in regulating inflammation is mediated through TGR5.

In the current study, we constructed a $K$. pneumoniaeinduced liver abscess and bacteremia mouse model with high reproducibility and stability, and comprehensively elucidated the protective effect of SBAs on the liver during bacterial infection by using multiomics and molecular experimental techniques. First, we used 16S rRNA sequencing technology to analyze the diversity of intestinal flora in the feces of mice with liver abscesses and healthy control mice, and found significant differences in intestinal flora composition between them. In addition, the development of liver abscess and bacteremia was found to lead to changes in specific intestinal flora, Ruminococcaceae, which specifically participate in the synthesis of SBAs. Secondly, through targeted metabolomics, we found that changes in intestinal flora composition led to significant changes in host metabolite abundance, with a decrease in the levels of LCA and DCA, and increase in those of cholic acid (CA). Based on these differences in SBAs, we found that LCA and DCA supplementation by gavage gains reduced organ bacterial loads and mortality. Furthermore, our results show that LCA and DCA mitigate inflammation in liver abscess models and reduce the expression of key cytokines and chemokines involved in inflammation by inhibiting the NF- $\kappa \mathrm{B}$ inflammatory signaling pathway. In addition, we found that SBAs significantly increase the expression of TGR5. In summary, our work highlights a potential role for SBA supplementation in reducing inflammation levels associated with KLA and bacteremia.

\section{Materials and Methods}

\section{Animals and Microorganisms}

Eight-week-old female C57BL/6J mice were purchased from Experimental Animal Center of Anhui Province (Hefei, China). All mice were maintained in a pathogenfree animal facility under a standard 12:12-h light/dark cycle. The animals were allowed free access to food and water at all times before the experiment. $K$. pneumoniae 
(serotypes $\mathrm{K} 1, \operatorname{mag} \mathrm{A}+, \operatorname{rmpA}+$, aerobactin + ) was isolated from patients with bacterial liver abscesses. The isolate was confirmed by time-of-flight mass spectrometry system (Clin-TOF-II, Beijing Yixin Bochuang Biotechnology Co., Ltd.). The strain was maintained by the Anhui Center for Surveillance of Bacterial Resistance (Hefei, China). K. pneumoniae was cultured in Luria broth (LB) at $37^{\circ} \mathrm{C}$ overnight. All experiments involving mice were performed in accordance with the guidelines of the Institutional Animal Care and Use Committee of Anhui Medical University (No. LLSC20190253).

\section{The K. pneumoniae-Induced Liver Abscess and Bacteremia Mouse Model and Treatment}

The mouse model was constructed by the following methods. The mice were anesthetized with isoflurane, and the abdomen was sterilized with $75 \%$ alcohol. The needle of a $1 \mathrm{~mL}$ syringe was inserted at an oblique angle $1-2 \mathrm{~cm}$ to the right of the midline of the abdomen of the mice. The needle was not inserted too deep, and the mice were confirmed to be infected after no blood was pumped back. During this period, mice were closely observed, and those that died within 24 hours were excluded.

The mice were randomly divided into six subgroups and given different treatments (group size is $8 \sim 10$ per group). Among these groups, the LCA group and DCA group were randomly selected and given LCA and DCA $(2.5 \mathrm{mg} / 10 \mathrm{~g}$ weight via oral gavage, for three consecutive days). Then, two randomly selected groups, designated the $\mathrm{KLA}+$ LCA group and KLA + DCA group, were respectively administered the same doses of LCA and DCA, and injected with $K$. pneumoniae $\left(10^{3} \mathrm{CFU} / \mathrm{mL}, 100 \mu \mathrm{L} / 10\right.$ $\mathrm{g}$ weight, via intraperitoneal injection) or the same amount of PBS after overnight fasting. Finally, the other two groups were gavaged with the same amount of corn oil for three days and then injected with $K$. pneumoniae or the same amount of PBS, and designated the KLA group and Control group, respectively. The dosage of $K$. pneumoniae and the selection of the dosage and supplementation method of SBAs used in this study were based on the previous study. $^{23,25,26}$ For survival curves, the general condition of mice were checked every $6 \mathrm{~h}$ until death occurred. Forty-eight hours after the K. pneumoniae injection, mice were killed, and feces, livers, and serum were extracted for further analysis.

\section{Targeted Metabolomics}

Targeted metabolomics analysis of bile acids from the feces and serum of KLA mice was performed at The Beijing Genomics Institute (BGI-Shenzhen, China). The extraction was performed according to the La Frano protocol. ${ }^{27}$ Briefly, $1 \mathrm{~mL}$ of cold methanol containing $10 \mathrm{~mL}$ of anti-oxidant solution was used, followed by the addition of each surrogate (SSTD, $250 \mathrm{nM}$ (deuterated bile acids for recovery calculations during data processing)). The homogenized solution was then centrifuged, dried, resuspended, and filtered using a $0.2 \mu \mathrm{m}$ PVDF membrane (Agilent 203980-100) for analysis with a liquid chromatograph-mass spectrometer (LC-MS). LC-MS analysis was performed on Acquity UPLC I-Class system with a Xevo TQ-S tandem-quadrupole mass spectrometer in multiplereaction monitoring mode (MRM) from Waters.

\section{DNA Extraction and I6S rRNA Sequencing}

Before mice were killed, fresh fecal samples were obtained, frozen, and stored at $-80^{\circ} \mathrm{C}$ immediately. A frozen aliquot (150 $\mathrm{mg})$ of each fecal sample was processed using the QIAamp Fast DNA Stool Mini Kit (QIAGEN). Next, 16S rRNA gene amplification, in vitro transcription, labelling, and hybridization were carried out as described previously. ${ }^{28}$ Diversity analysis and taxonomy-based analysis were performed at 97\% similarity level using mothur (http://www.mothur.org) ${ }^{29}$ as described in previous studies. ${ }^{30,31}$ The Mann-Whitney $U$-test was performed using SPSS 16.0 software.

\section{Analysis of the Bacterial Burden}

Blood, liver and spleen tissues of each group were collected before euthanasia. To perform blood CFU counts, sterile phosphate-buffered saline (PBS) was used and dilutions were plated on nutrient agar plates. The liver tissue samples were weighed and collected in a $1.5 \mathrm{~mL}$ EP tube containing pre-cooled PBS. Samples were homogenized for $30 \mathrm{~s}$ on a grinder (Tissuelyser-32, Shanghai JinXin Industrial Development CO., LTD, Shanghai, China), followed by continuous dilution and plating on nutrient agar plates. $\mathrm{CFU}$ numbers were counted after overnight incubation at $37^{\circ} \mathrm{C}$.

\section{Histopathological Examination and TUNEL Staining}

The liver tissue was fixed in $10 \%$ buffered formalin and processed for hematoxylin and eosin (H\&E) analysis. The 
degree of liver inflammation was determined by a blinded histopathology score, as described previously. ${ }^{32,33}$ A TUNEL bright green apoptosis detection kit from Vazyme (Nanjing, China) was used for detection of apoptotic cells in the liver tissue sections, according to the manufacturer's instructions. Cells were observed under a fluorescence microscope (Olympus, Tokyo, Japan).

\section{Immunohistochemistry (IHC)}

Livers were fixed with $10 \%$ buffered formalin and embedded in paraffin according to the standard protocol. Paraffinembedded liver tissue was cut into 5 - $\mu \mathrm{m}$-thick sections. After dewaxing and hydration, antigen retrieval was conducted with $0.01 \mathrm{M}$ sodium citrate buffer solution ( $\mathrm{pH}$ 6.0). Sections were incubated with anti-p-IкB $\alpha$ (1:200 dilution, Bioss, catalog [cat.] no. 5515R), anti-p-p50 (1:200 dilution, Bioworld, cat. no. 4132), anti-p-p65 (1:200 dilution, Santa Cruz, cat. no. 136548), and F4/80 (1:100 dilution, Abcam, cat. no. 111101) monoclonal antibodies at $4^{\circ} \mathrm{C}$ overnight. The color reaction was developed with an HRP-linked polymer detection system and counterstaining with hematoxylin was performed before dehydrating in graded alcohols. F4/80, TGR5-, p-IкB $\alpha-$, p-p50-, and p-p65-positive cells were counted in ten randomly selected fields from each slide, at a magnification of $\times 200$.

\section{Analysis of Biochemical Parameters}

Examinations of plasma IL-6, MCP-1, and IL-10 were performed using a mouse IL-6 ELISA kit (cat. no. JYM0012Mo), a mouse MCP-1 ELISA kit (cat. no. JYM0099Mo), and a mouse IL-10 ELISA kit (cat. no. JYM0005Mo) according to the manufacturer's protocol (Wuhan Genemei Biotechnology Co., Ltd., Wuhan, China). Plasma alanine aminotransferase (ALT) and aspartate aminotransferase (AST) were assayed using an alanine aminotransferase assay kit (cat. no. C009-2-1) and aspartate aminotransferase assay kit (cat. no. C010-2-1), respectively (Jian Cheng Bioengineering Institute, Nanjing, China).

\section{RNA Extraction from Tissues and Quantification by RT-PCR}

Total RNA was extracted from liver tissues using TRIzol Reagent (Invitrogen). RNase-free DNase-treated total RNA (500 ng) was reverse-transcribed using AMV reverse transcriptase (Promega). Real-time RT-PCR was performed to quantify mRNAs of inflammatory cytokines and chemokines in the liver. Specific primers for the amplified genes are listed in Table 1. The amplification reactions were carried out on a LightCycler 480 Instrument (Roche Diagnostics $\mathrm{GmbH}$ ) with an initial hold step $\left(95^{\circ} \mathrm{C}\right.$ for $\left.5 \mathrm{~min}\right)$ and 50 cycles of a three-step PCR $\left(95^{\circ} \mathrm{C}\right.$ for $15 \mathrm{~s}, 60^{\circ} \mathrm{C}$ for $15 \mathrm{~s}$, and $72^{\circ} \mathrm{C}$ for $30 \mathrm{~s})$.

\section{Western Blotting}

For Western blotting, liver tissue samples $(100 \mathrm{mg})$ were added to RIPA cell lysate (containing 1mM PMSF) and lysed on ice for $30 \mathrm{~min}$. The supernatant obtained after centrifugation at $12,000 \mathrm{rpm}$ for 10 minutes contained the total tissue protein. The extraction of liver nuclear proteins was performed based on previous studies. ${ }^{34}$ Then, $3 \times$ SDSPAGE protein loading buffer was added to the collected protein samples at 1:2, and the solution was heated in a boiling water bath for 10 minutes to fully denature the protein. After cooling to room temperature, the protein sample was directly loaded on to a SDS-PAGE gel. The samples were then subjected to electrophoresis, membrane transfer, and sealing. For total proteins, the membrane was incubated overnight with the following antibodies: TGR5 (1:5000 dilution, Abcam72608), p-IкB $\alpha$ (1:1000 dilution, Bioss, cat. no. 5515R), ІкB $\alpha$ (1:2000 dilution, Abcam, catalog cat. no. 32518), and $\beta$-actin (1:1000 dilution, Abcam, cat. no. 8227). For the nucleoprotein, the membrane was incubated overnight with antibodies p-p65 (1:1000 dilution, Santa Cruz, cat. no. 136548), p-p50 (1:1000 dilution, Bioworld, cat. no. 4132), and LaminB1 (1:500 dilution, Bioss, cat. no. 33040M). Finally, an ECL luminescence kit was used to detect signals according to the manufacturer's instructions.

Table I Primers for Real-Time RT-PCR

\begin{tabular}{|l|l|l|}
\hline Genes & \multicolumn{1}{|c|}{ Forward $\left.\mathbf{( 5}^{\prime} \mathbf{-} \mathbf{3} \mathbf{\prime}\right)$} & \multicolumn{1}{c|}{ Reverse (5'-3') } \\
\hline GAPDH & AGGTCGGTGTGAACGGATTTG & GGGGTCGTTGATGGCAACA \\
$M C P-I$ & TAAAAACCTGGATCGGAACCAAA & GCATTAGCTTCAGATTTACGGGT \\
$K C$ & ACTGCACCCAAACCGAAGTC & TGGGGACACCTTTTAGCATCTT \\
$I P-10$ & CCAAGTGCTGCCGTCATTTTC & GGCTCGCAGGGATGATTTCAA \\
TGF- $\beta$ & CCACCTGCAAGACCATCGAC & CTGGCGAGCCTTAGTTTGGAC \\
\hline
\end{tabular}




\section{Statistical Analysis}

The sample distribution was determined by the normality test. Normally distributed variables are presented as the mean $\pm \mathrm{S}$.

E.M. Two sample $t$-test was used to determine differences between KLA mice and healthy controls. Differences between three or more groups were evaluated using oneway analysis of variance (ANOVA). For the nonparametric tests, the two-tailed Mann-Whitney $U$-test was performed to evaluate statistical differences between two groups. The level of statistical significance was set at a two-tailed $P$-value of 0.05. GraphPad Prism version 7.0 (GraphPad Software) and SPSS version 16.0 were used for statistical analyses.

\section{Results}

\section{Comparison of Absolute Concentration of SBAs Between KLA Mice and Healthy Controls}

To better understand the potential role of SBAs in bacterial liver abscesses, we first conducted a targeted metabolomics study using LC-MS to assess the differences in LCA and DCA in feces and serum between KLA mice and healthy controls. LCA and DCA in both serum (Figure 1A) and feces (Figure 1B) were significantly reduced in KLA mice compared with that in healthy controls.

A

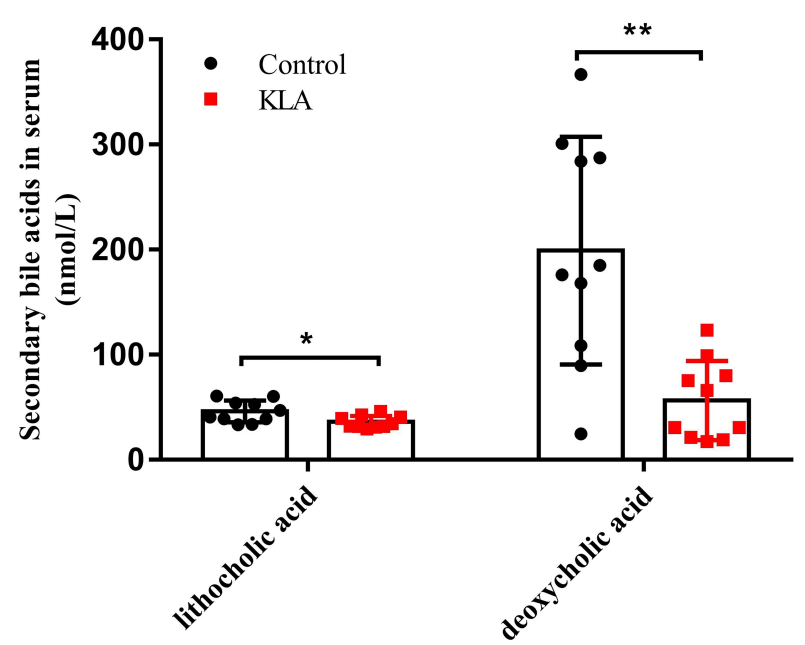

\section{Alterations of Overall Structure and Composition of the Gut Microbiota After}

\section{K. pneumoniae Infection}

To determine whether the changes in the levels of circulating metabolites were correlated with altered abundances of specific intestinal flora, we performed 16S rRNA sequencing of V4 variable regions. We identified the differences by analyzing the composition of intestinal bacteria between KLA mice and healthy controls. We obtained $2,015,829$ high-quality reads from pyrosequencing analysis, of which 3,018,106 sequences were derived from KLA mice and 1,013,552 from healthy controls. To determine the number of strains or genera from the sequencing results, it is necessary to classify the sequences, generally clustering those with a similarity of more than $97 \%$ as an OTU. Figure $2 \mathrm{~A}$ shows the OTU species accumulation curve, which is widely used to determine the adequacy of sample size and estimate species richness. As the number of sampled individuals increases, the curve tends to become flat, indicating that the sample size has met or even exceeded the experimental requirements. The Venn diagram shows the number of overlapping and specific OTUs between two groups. A total of 13,303 OTUs were obtained from the samples; among these, 601 OTUs were shared between healthy and KLA groups, with 12
B

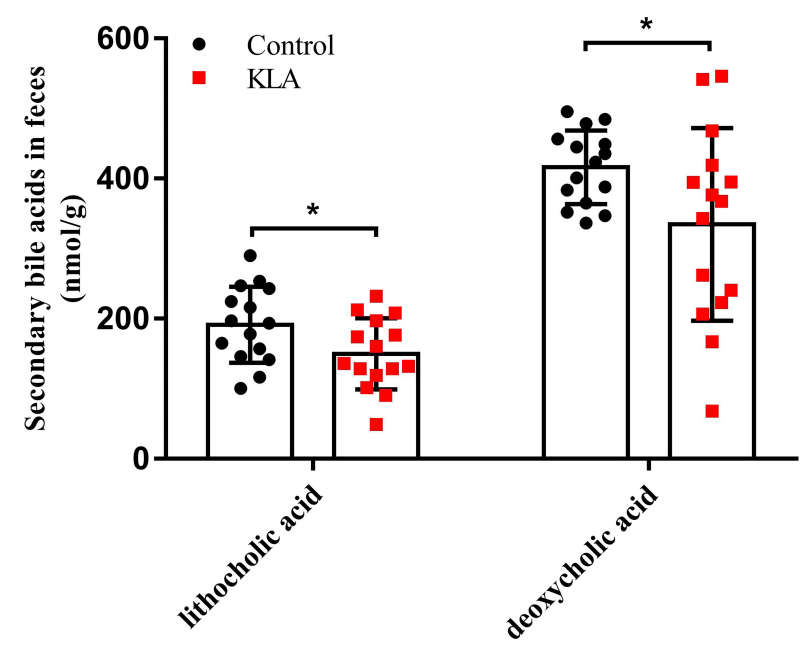

Figure I Analysis of absolute concentration of bile acids in KLA mice and healthy controls. Levels of lithocholic acid and deoxycholic acid in the feces (A) and serum (B) of were detected in mice with KLA and in the healthy controls. All data were expressed as means \pm S.E.M. $(N=10-15)$; $* P<0.05$, $* * P<0.01$ as compared with that in healthy controls. 
A

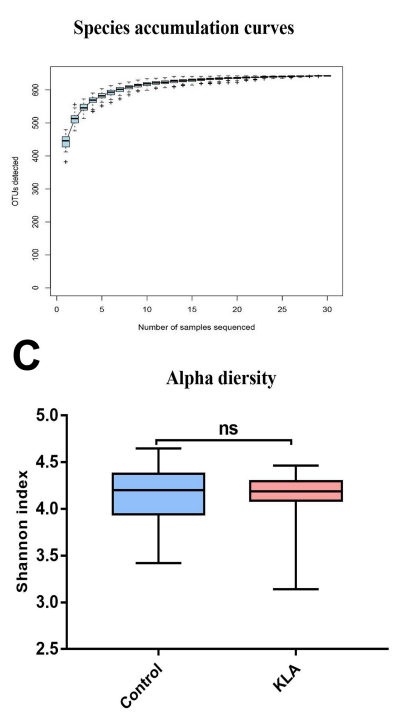

B

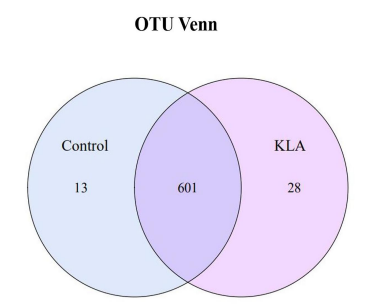

D

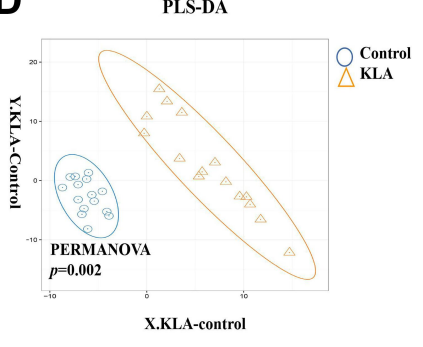

E
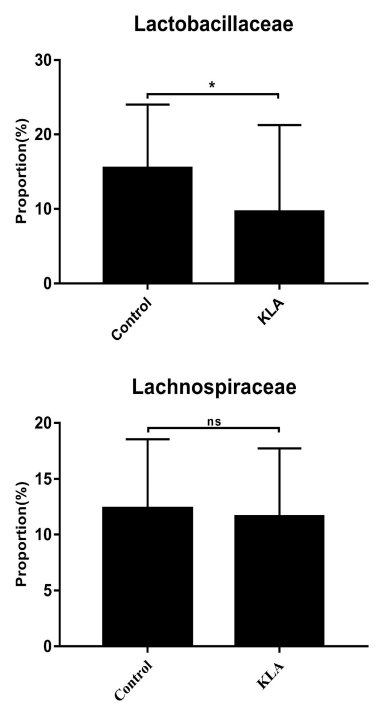

Ruminococcaceae
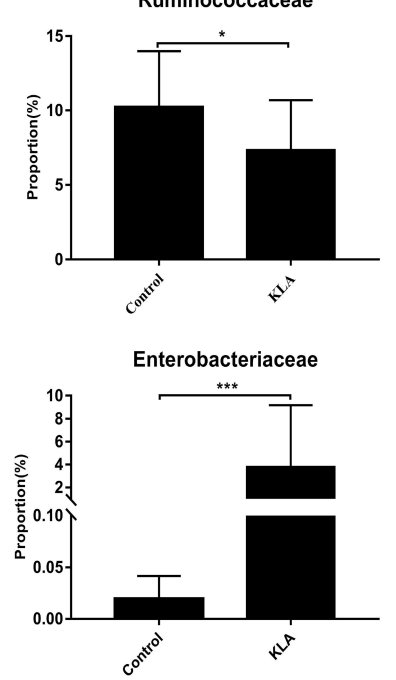

Figure 2 Overall structure of microbiota in KLA mice and controls. (A) The species accumulation curve (smooth tendency) shows sufficient sample numbers in a boxplot of the observed species. The $\mathrm{X}$-axis indicates the number of samples and the Y-axis shows the observed species (OTU number). (B) The OTU Venn diagram shows the OTUs that overlap in KLA mice and healthy controls. (C) Alpha diversity (Shannon index) of I6S rRNA genes from KLA mice and healthy controls. (D) PLS-DA score plot of species abundance in samples from individuals with KLA and healthy controls. PERMANOVA with the Bray-Curtis distance was used to assess the significance of differences between the two groups $(P=0.002)$. (E) Abundance of discriminative OTUs in the taxonomic distributions at the family levels between KLA mice and healthy controls. $P$-values are determined by two-tailed Wilcoxon rank-sum test $(\mathbf{C})$ and two-sample $t$-test $(\mathbf{E})(\mathrm{N}=15)$; $* P<0.05, * * * P<0.00 \mathrm{I}$ as compared with healthy controls.

and 30 specific OTUs, respectively (Figure 2B). Although the intestinal flora of $K$. pneumoniae-infected mice showed no difference in Shannon diversity index ( $\alpha$-diversity) (Figure 2C), partial least squares-discriminant analysis (PLS-DA) (Figure 2D) revealed a distinct clustering pattern between samples from individuals with KLA and healthy controls, indicating that the composition of the intestinal flora in mice with liver abscesses had been substantially remodeled. These results indicate that intraperitoneal injection of $K$. pneumoniae altered fecal microflora composition, although there was no substantial difference in diversity between the KLA group and the control group. Next, we analyzed the composition of the cecal microflora between the healthy and KLA mouse groups. In all identified taxa, we observe a significant reduction in the Ruminococcaceae and Lactobacillaceae families; in contrast, an increase in harmful bacteria associated with enterohepatic disorders was found, indicating that disordered intestinal flora may be a contributing factor to KLA progression (Figure 2E). Interestingly, Ruminobacteriaceae and Lachnospiraceae comprise few bacteria known to be involved in SBA synthesis, which perform subsequent CA and CDCA 7A-dehydroxylation reactions to produce DCA and LCA, respectively. In fact, a reduction was only found in the Ruminococcaceae family, while no differences in
Lachnospiraceae were observed between the two groups (Figure 2E).

\section{Effects of SBAs on the KLA Mice}

As shown in Table 2, KLA mice were given SBAs by intragastric administration. The results showed that the mice with KLA pretreated with either LCA or DCA regained weight after 48 hours; further, these mice were less likely to develop visible abscesses in the liver. Moreover, in terms of preventing abscesses, DCA was more effective than LCA. In addition, the relative liver weights of $K$. pneumoniae-infected mice treated with DCA were slightly lower than those of untreated mice; however, this effect was not observed with LCA.

\section{Effects of SBAs on K. pneumoniae-Infected Mice}

We further observed the effect of SBAs on KLA by evaluating the survival, bacterial counts, liver pathology, and immunohistochemistry. Before the $K$. pneumoniae challenge, mice were administered DCA and LCA for 3 days. SBAs reduced susceptibility to $K$. pneumoniae infections, as demonstrated by the decreased bacterial burdens and increased survival rates (Figure 3A-C and $\underline{\mathrm{S} 1}$ ). In addition, exogenous supplementation with gut microbiota-derived SBAs elicited 
Table 2 Body Weight and Liver Weights

\begin{tabular}{|l|c|c|c|c|}
\hline & $\begin{array}{c}\text { Number } \\
\text { of Liver } \\
\text { Abscesses }\end{array}$ & $\begin{array}{c}\text { Body } \\
\text { Weight (g) }\end{array}$ & $\begin{array}{c}\text { Absolute } \\
\text { Liver } \\
\text { Weight } \\
\text { (g) }\end{array}$ & $\begin{array}{c}\text { Relative } \\
\text { Liver Weight } \\
\text { (\%) }\end{array}$ \\
\hline KLA & $10 / 10$ & $21.87 \pm 1.25$ & $1.05 \pm 0.06$ & $0.048 \pm 0.004$ \\
KLA+LCA & $7 / 10$ & $22.99 \pm 1.06^{*}$ & $1.05 \pm 0.11$ & $0.045 \pm 0.004$ \\
KLA+DCA & $3 / 10$ & $23.00 \pm 0.84^{*}$ & $1.01 \pm 0.04$ & $0.044 \pm 0.002^{*}$ \\
\hline
\end{tabular}

Notes: Data were expressed as means \pm S.E.M. $(\mathrm{N}=10) ; * P<0.05$ as compared with the Control group.

significant improvement in liver histopathology in K. pneumoniae-infected mice (Figure 3D). The liver injury induced by bacterial infection was further confirmed by TUNEL assays. Compared with that in untreated KLA mice, the TUNEL-positive percentage was reduced in the liver of KLA mice after gavage with SBAs (Figure 3E). We then performed immunohistochemical staining for $\mathrm{F} 4 / 80$, a mature tissue macrophage marker, on the liver samples to assess Kupffer cell infiltration. The number of F4/80-positive cells in the KLA mice was higher than in the healthy controls (Figure 3F). After SBAs were administered, the number of F4/80-positive cells in the liver of KLA mice was significantly reduced. The results showed that SBAs reduced the infiltration of Kupffer cells. In conclusion, the above results highlight the protective role of SBAs in the liver.

\section{SBAs Ameliorated Inflammation Levels in KLA Mice}

As shown in Figure 4A, mRNA expression levels of proinflammatory factors and chemokines, such as tumor necrosis factor (TNF)-a, monocyte chemokine (MCP)-1, keratinocyte chemokine (KC), and interferon-induced protein (IP)-10 in the livers of the KLA group were compared with those of healthy controls. KLA mice pretreated with SBAs (DCA and LCA) showed reduced levels of inflammation and chemokine transcription in the liver (Figure 4A). Furthermore, the serum levels of interleukin (IL)-6 and MCP-1 in the drug-treated KLA mice were significantly lower than in the untreated

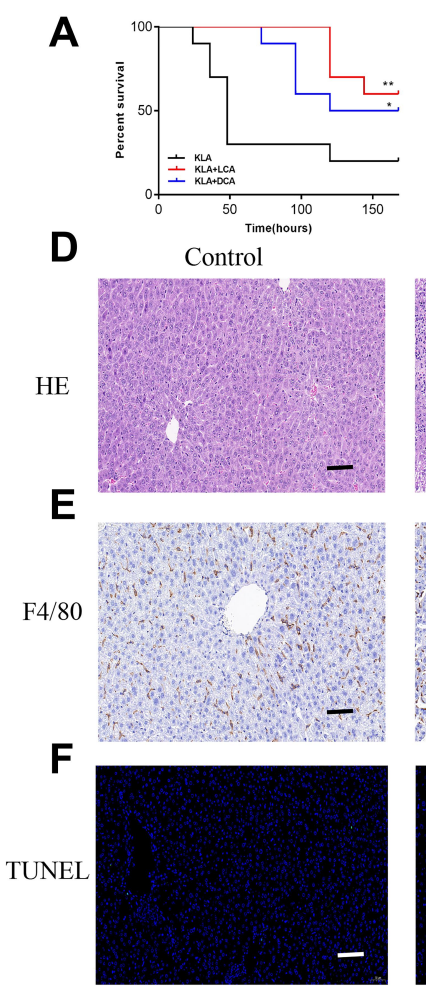

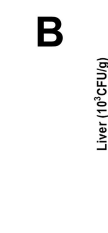
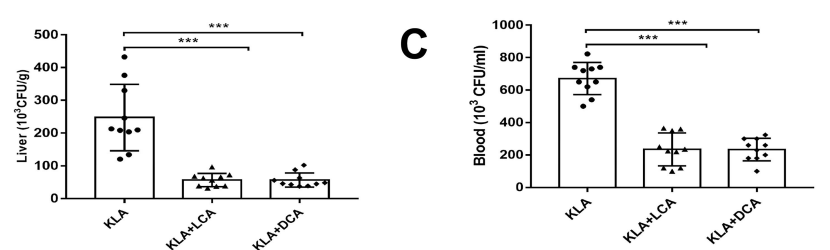

KLA

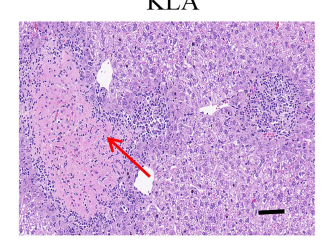

$\mathrm{KLA}+\mathrm{LCA}$
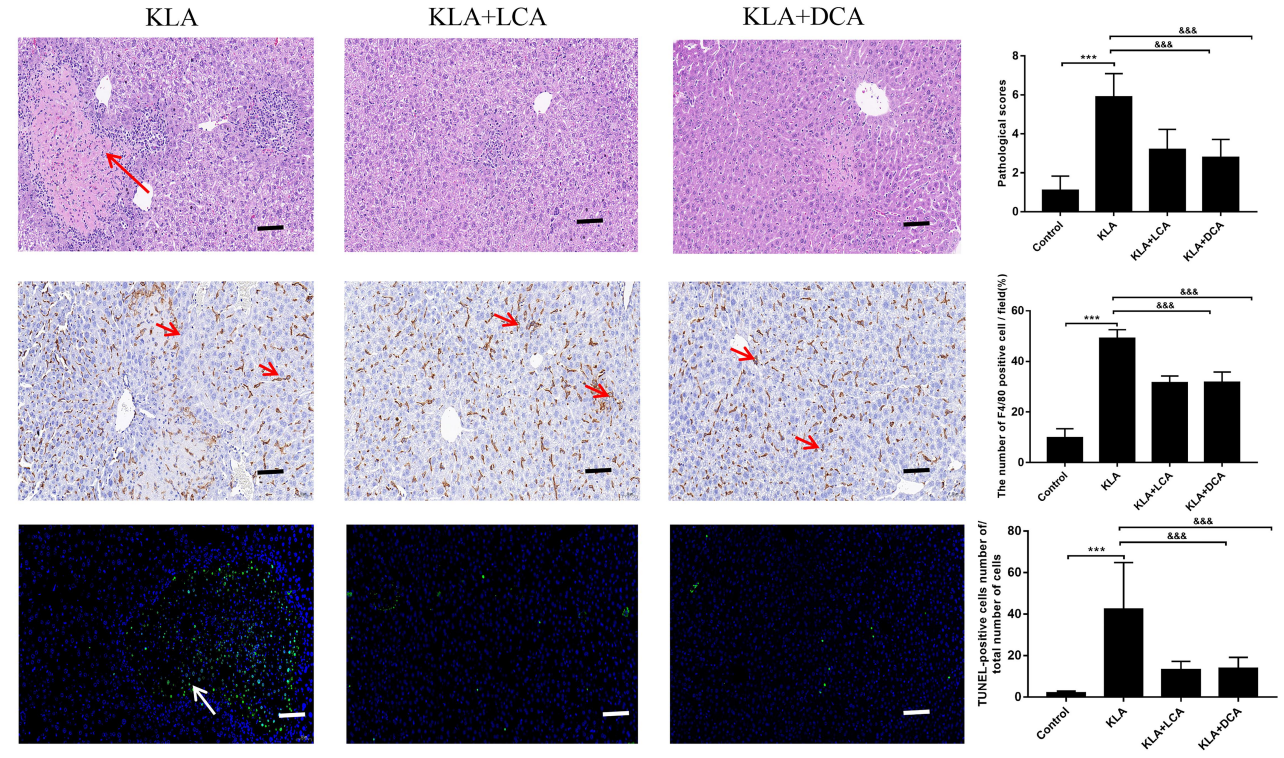

Figure 3 Effects of SBAs on K. pneumoniae-induced liver abscess. (A) Kaplan-Meier survival plot of SBA-treated and untreated mice after $K$. pneumoniae infection. (B and C) CFUs of $K$. pneumoniae in blood (B) and liver (C) at $48 \mathrm{~h}$ after infection in SBA-treated and untreated mice. (D) Liver abscess and necrosis were examined by hematoxylin and eosin staining and observed under a microscope (magnification, $\times 200$ ) in SBA-treated and untreated mice $48 \mathrm{~h}$ after $K$. pneumoniae infection. The red arrowheads indicate liver abscess. KLA mice show increased histologic evidence of liver abscess and necrosis, as indicated by pathological scores, following $K$. pneumoniae challenge. (E) Representative TUNEL staining of sections from livers of SBA-treated and untreated mice (magnification, $\times 200$ ) and statistical analysis of the number of TUNEL-positive cells per total number of cells. The white arrowheads indicate apoptotic hepatocytes. The number of cells in at least 10 microscopic fields was counted. (F) Representative immunohistochemistry staining for F4/80 from livers of SBA-treated and untreated mice (magnification, $\times 200$ ) and statistical analysis of the number of F4/80-positive cells per field. The red arrows indicate F4/80 positive cells. Cells in eight high-power fields were counted. All data are expressed as means \pm S.E.M ( $n=8-10$ ); $* P<0.05$, **P $<0.01$, $* * * P<0.001$ as compared with healthy controls; ${ }^{\text {\&\&\& } P}<0.001$ as compared with KLA mice. 
group, while those of IL-10, which is known inhibit inflammatory responses, were increased. The levels of cytokines in mouse serum were measured using ELISA, see supporting Figure 4B. In KLA mice treated with SBAs, levels of ALT and AST, two markers of liver injury, were significantly reduced (Figure 4C). These results indicate that SBAs can inhibit the inflammatory response induced by KLA.

\section{Effects of SBAs on NF- $\kappa$ B Signaling in the Livers of $K$. pneumoniae-Infected Mice}

The effect of SBAs on NF- $\mathrm{KB}$ signaling in the liver in response to bacterial infection was analyzed. As expected, the level of $\mathrm{p}-\mathrm{I} \kappa \mathrm{B} \alpha$ protein in the cytoplasm was increased $48 \mathrm{~h}$ after $K$. pneumoniae intraperitoneal injection; in addition, significantly higher levels of the nuclear protein p-p65 and p-p50 subunits were found (Figure 5A-E). The levels of three proteins, namely $\mathrm{p}-\mathrm{I} \kappa \mathrm{B} \alpha$, nuclear $\mathrm{p}-\mathrm{p} 65$, and $\mathrm{p}$-p 50 , were all markedly decreased in SBAs-treated KLA mice compared with those in controls. Immunohistochemistry also showed that the number of hepatic p-IкB $\alpha$-, nuclear NF-kB p65-, and p50-positive cells were significantly increased in KP-treated mice (Figure $5 \mathrm{~F}-\mathrm{I}$ ). The number of $\mathrm{p}-\mathrm{I} \kappa \mathrm{B} \alpha-$, nuclear p-p65-, and p-p50-positive cells in the liver were significantly reduced in SBA-treated KLA mice compared with that in untreated KLA mice (Figure 5F-I).

\section{Effects of SBAs on Hepatic TGR5 Signaling}

The effect of SBAs on hepatic TGR5 activity was analyzed. The expression of TGR5 mRNA was up-regulated by SBAs compared with the control group (Figure 6A), and the relative level of TGR5 protein was significantly increased in SBA-treated mice (Figure 6B and C).

\section{Discussion}

Intraperitoneal infection of hypervirulent $K$. pneumoniae can prone to developing liver abscess and bacteremia, complications such as necrotizing fasciitis, endophthalmitis and disseminated meningitis through bloodstream dissemination. Furthermore, the high morbidity and mortality associated with liver abscesses and bacteremia make this disease a lifethreatening and difficult clinical problem. ${ }^{35}$ In this study,
A

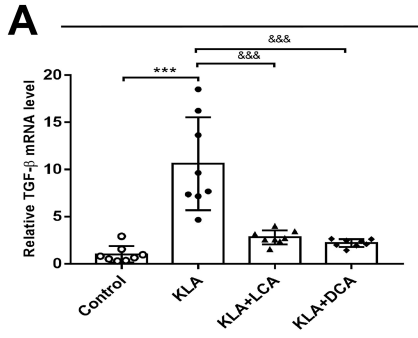

B
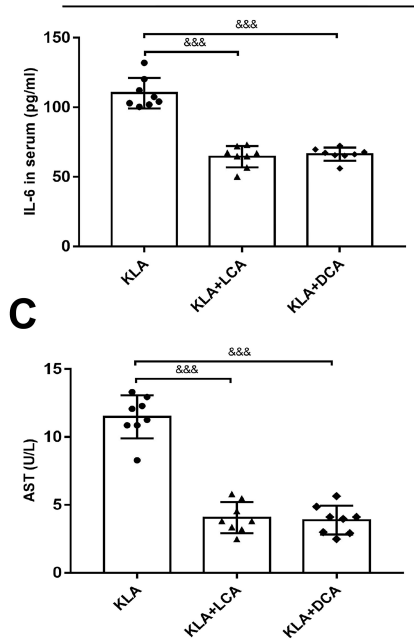

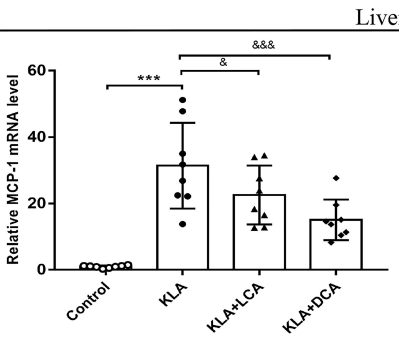

Liver
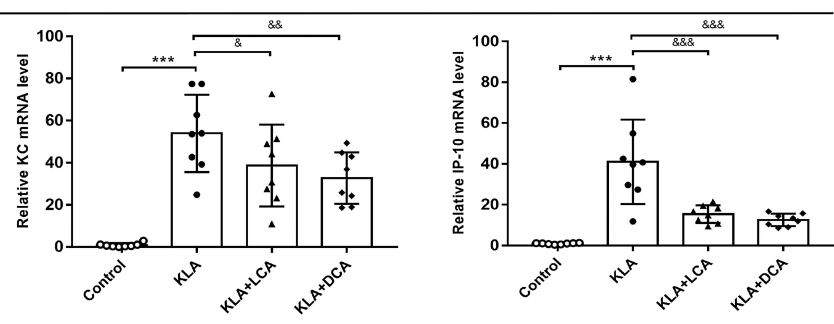

Serum
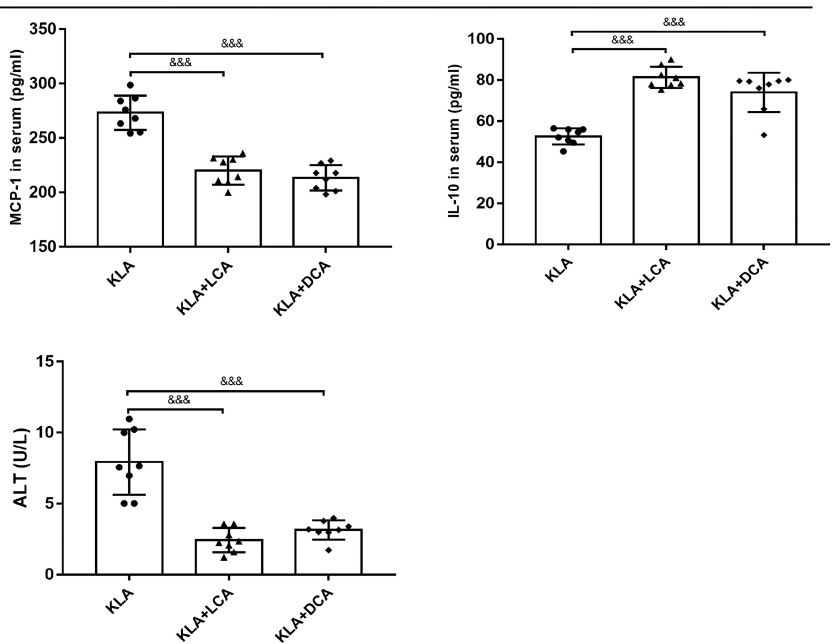

Figure 4 SBAs ameliorate inflammation associated with $K$. pneumoniae-induced liver abscess in. (A) Real-time RT-PCR analysis of TGF- $\beta$, MCP-I, KC, and IP-I0 mRNAs expression in four different groups. (B and C) The levels of inflammatory serum markers (IL-6, MCP-I, and IL-I0) (B), ALT, and AST (C) in serum from KLA mice that were treated with corn oil, LCA, or DCA. All data are expressed as means \pm S.E.M $(n=8),{ }^{* * *} P<0.001$ as compared with healthy mice; ${ }^{\&} P<0.05$, ${ }^{\& \& \&} P<0.00 \mathrm{I}$ as compared with KLA mice. 
A

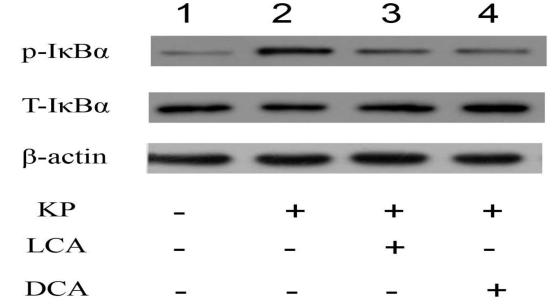

B

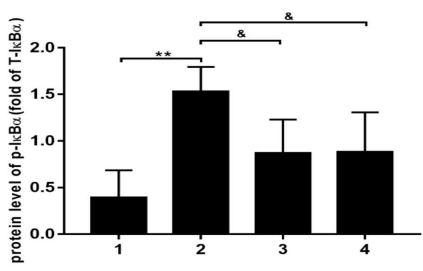

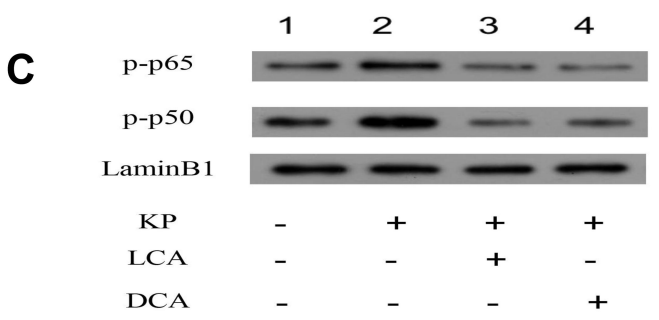

$\mathbf{F}$

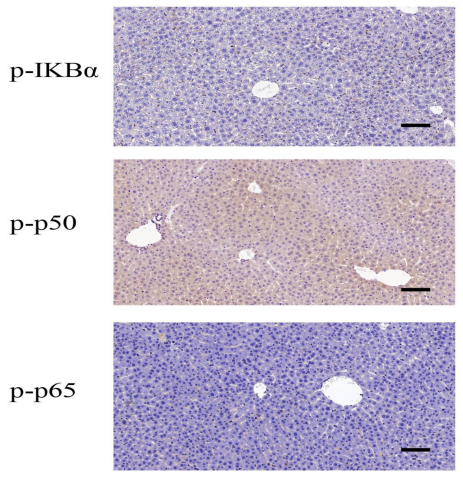

G

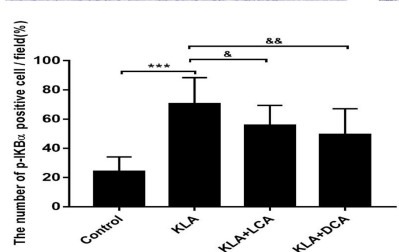

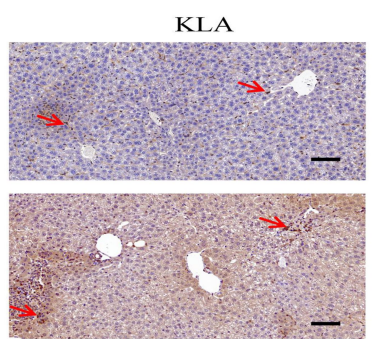

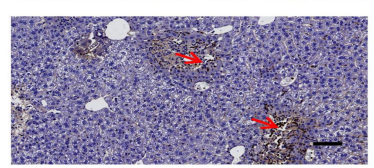

H

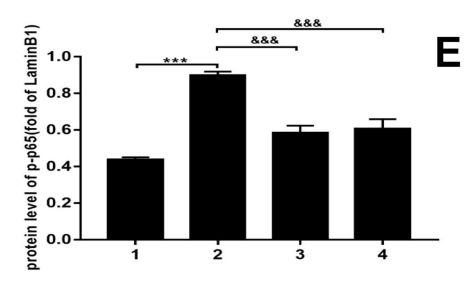

$\mathrm{KLA}+\mathrm{LCA}$
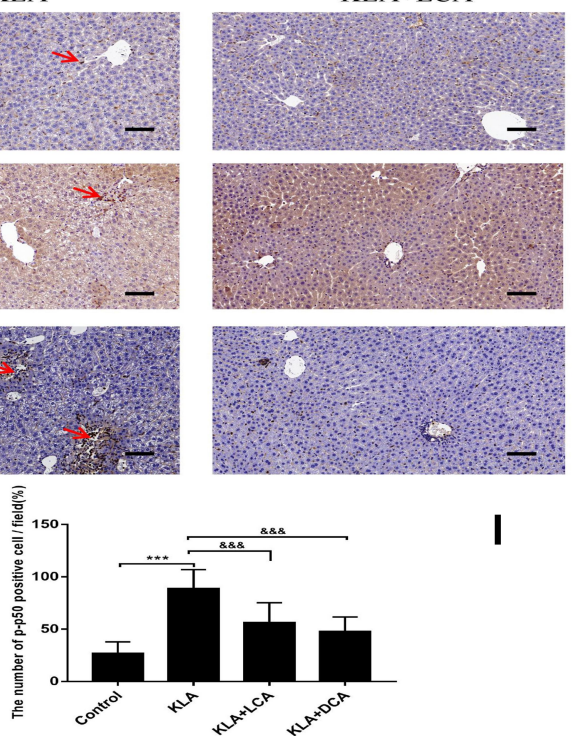

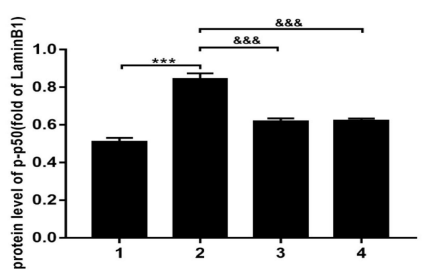

$\mathrm{KLA}+\mathrm{DCA}$
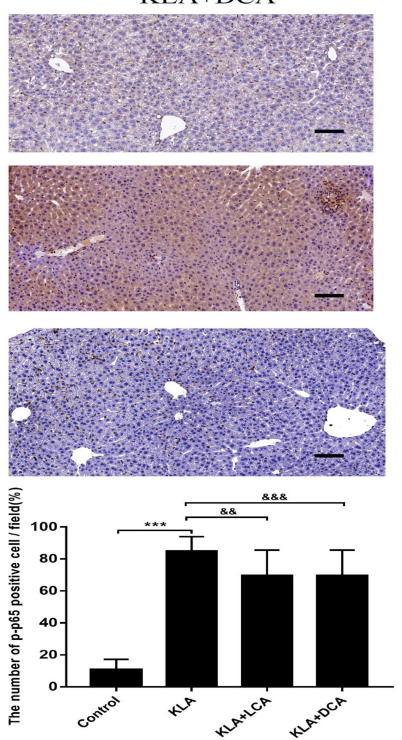

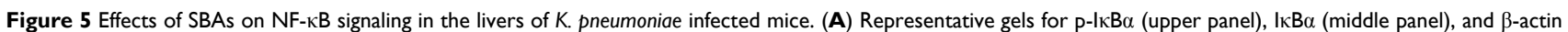
(lower panel) are shown. (B) Quantitative analysis of $\mathrm{p}-\mathrm{l} \kappa \mathrm{B} \alpha$ was performed by scanning densitometry. (C) Representative gels for p-p65 (upper panel), $\mathrm{p}-\mathrm{p} 50$ (middle panel), and LaminBI (lower panel) are shown. (D) Quantitative analysis for p-p65 was performed by scanning densitometry. (E) Quantitative analysis for p-p50 was performed by scanning densitometry. (F) Representative photomicrographs of liver $\mathrm{p}-\mathrm{l} \kappa \mathrm{B} \alpha, \mathrm{p}-\mathrm{p} 50$, and $\mathrm{p}-\mathrm{p} 65$ are consecutively shown. Original magnification: $200 \times$. The red arrowheads indicate $\mathrm{p}$-I $\mathrm{k} \mathrm{B} \alpha$, nuclear $\mathrm{p} 50$, and $\mathrm{p} 65$-positive cells, respectively. $(\mathbf{G}) \mathrm{p}$-I $\mathrm{k} \mathrm{B} \alpha$-positive cells were compared between different groups. $(\mathbf{H}) \mathrm{Nuclear} p 50$-positive cells were compared between different groups. (I) Nuclear p65-positive cells were compared between different groups. All experiments were duplicated for three times. Data are expressed as means \pm S.E.M $(n=10) ;{ }^{* * P}<0.01$, ***P $<0.001$ as compared with healthy controls; ${ }^{\&} P<0.05,{ }^{\& \&} P<0.01$, ${ }^{\& \&} P<0.001$ as compared with KLA mice.

targeted metabolomics and 16S rRNA sequencing showed that the structure of the intestinal flora and the level of SBAs were significantly altered in K. pneumoniae-infected mice compared with those in healthy control mice. Pretreatment with SBAs significantly improved the survival, organ bacterial loads, liver pathology, and immunohistochemistry of K. pneumoniae-infected mice. In addition, SBAs play an antiinflammatory role by inhibiting the NF- $\mathrm{KB}$ inflammatory signaling pathway in the liver via TGR5 receptor activation. Studies have also found that the intestinal flora affects the host immune response mainly by regulating the synthesis of intestinal flora metabolites. ${ }^{36,37}$ Diverse microorganisms comprise the intestinal ecosystem; ${ }^{36}$ their metabolites, eg, bile acids, aromatic amino acids, and short-chain fatty acids, play important roles in signaling, thus modulating host metabolism and inflammation by activating the corresponding receptors. $^{38-40}$ Among them, SBAs are closely related to the liver through the gut-liver axis, transported from the gut back to the liver via the enterohepatic circulation. ${ }^{41}$ In summary, the novel insights gleaned from this study on the role of the intestinal flora and their metabolites is of significance for the treatment of bacterial infections of the liver. 
A

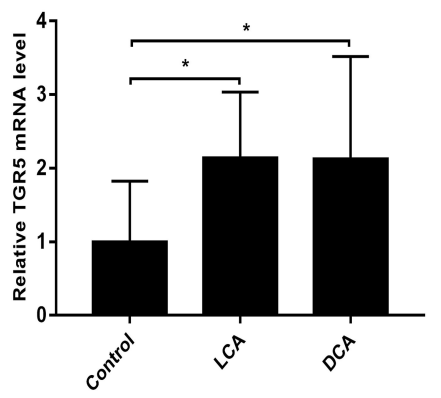

B

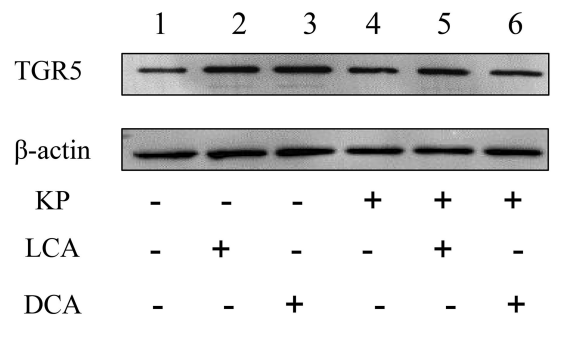

C

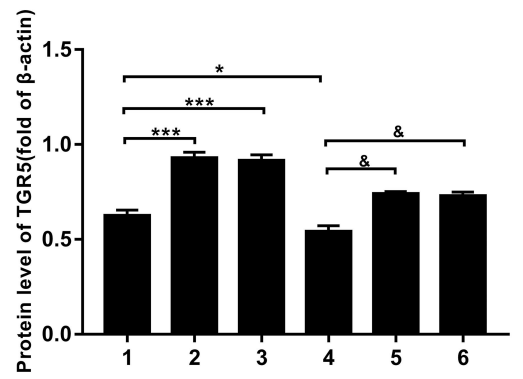

Figure 6 Effects of SBAs on hepatic TGR5 signaling. (A) Hepatic TGR5 mRNA was measured by real-time RT-PCR. (B) Representative gels for TGR5 and $\beta$-actin are shown. (C) Quantitative analysis of TGR5 was performed by scanning densitometry. All experiments were duplicated for three times. Data are expressed as means \pm S.E.M ( $\mathrm{n}=10$ ); $* P<0.05$, $* * * P<0.001$ as compared with healthy controls; ${ }^{\circledR} P<0.05$ as compared with KLA mice.

SBAs are originally derived through cholesterol metabolism in the liver, and are further metabolized by the intestinal flora; their production is dependent on the gutliver feedback loop. ${ }^{42}$ Most bile acids are reabsorbed in the distal small intestine, with the remaining bile acids entering the colon at concentrations of about $200 \mu \mathrm{M}$ to $1000 \mu \mathrm{M}$. Among the various metabolites in the normal intestinal microenvironment, SBAs are present in the highest concentrations. ${ }^{17}$ We detected the content of DCA and LCA in the fecal samples by using multiple reaction monitoring technology and found that the concentration of SBAs was as high as $568.1 \mathrm{nmol} / \mathrm{g}$, and that the average concentration was in the top three among multiple indicators. In the host intestinal tract, only members of the Ruminococcaceae and Lachnospiraceae families, with relatively rare 7 - dehydroxylation ability, participate in the complex process of PBA conversion into SBAs, with important implications for regulating the activity of the immune system. ${ }^{42}$ In ulcerative colitis patients, a significant lack of SBAs has been observed in the pouch after colectomy, which was consistent with the reduction in abundance of Clostridium leptum, a more homologous family member of the Ruminococcaceae family. ${ }^{25}$ Our data show that the intestinal bacterial composition of mice was significantly altered in the process of liver abscess formation in K. pneumoniae-infected mice, which was specifically reflected by the increase in Enterobacteriaceae and the significant decrease in Lactobacillus. Moreover, the Ruminococcaceae was also significantly decreased compared with that in healthy mice. Many studies have shown that the structural alterations in the intestinal flora can lead to profound changes in the levels of host metabolites. Our targeted metabolomics results also supported this view: DCA and LCA in both serum and feces were low in $K$. pneumoniae-infected mice, which might be related to the reduction of Ruminococcaceae caused by bacterial infection.

These findings raise the question of whether it is possible to correct physiological disorders in the host by restoring key metabolites. In a previous study, SBAs were shown to act as an antimicrobial agent with direct effects on microorganisms: DCA and LCA were demonstrated to destroy bacterial cell membrane integrity, induce DNA damage, or inactivate proteins via denaturation, thereby inhibiting the growth of intestinal pathogenic bacteria and reducing their colonization of the susceptible host. ${ }^{43}$ SBAs can also indirectly inhibit the growth and proliferation of intestinal bacteria by regulating host immunity to induce the production of antimicrobial peptides or stimulate production of factors such as nitric oxide synthase and interleukin by the intestinal epithelium. In the current study, we found that supplementation with SBAs (LCA and DCA), a metabolite derived from intestinal flora, reduced susceptibility to $K$. pneumoniae infection, as demonstrated by the reduction in bacterial burden and improvement in survival rates.

SBAs are rapidly emerging as promising agents that modulate the expression of inflammatory factors. This study investigated the effects of SBAs on systemic and local inflammatory cytokines in liver abscess and bacteremia. First, inflammatory responses were observed in the K. pneumoniae-infected mice. We found that the hepatic local immune system was activated in response to K. pneumoniae challenge, and that the population of F4/80positive Kupffer cells was higher than in the healthy controls. However, we found that it was reported the absolute numbers of Kupffer cells were quite constant except for a small increase during the $12 \mathrm{~h}$ and $24 \mathrm{~h}$ time points. ${ }^{44}$ Different 
results may be related to different modeling methods, observation time points and experimental techniques. The current study also found that macrophagic infiltration promotes the rapid release of TNF- $\alpha$, MCP-1, KC, and IP-10, amplifying the inflammatory cascade, finally leading to inflammatory storms. These results suggested that liver abscesses cause a certain degree of immune inflammation. ${ }^{44}$ An increase in ALT and AST, two sensitive markers indicating liver injury, was detected in $K$. pneumoniae-infected mice. It is worth mentioning that the levels of $\mathrm{p}-\mathrm{I} \kappa \mathrm{B} \alpha$, nuclear NF- $\kappa \mathrm{B}$ p65, and p50 subunits in the liver were significantly increased, suggesting the activation of the NF- $\kappa$ B pathway. ${ }^{45,46}$ Second, the elevation in the above indicators (macrophage infiltration, inflammatory cytokines, chemokines, and liver function) in KLA mice were ameliorated in SBA-treatment $K$. pneumoniae-infected mice. We also found that the phosphorylation of $\mathrm{p}-\mathrm{I} \kappa \mathrm{B} \alpha, \mathrm{p}-\mathrm{p} 65$, and $\mathrm{p}$-p50, three key proteins of the NF- $\kappa \mathrm{B}$ inflammatory signaling pathway, was alleviated in SBA-treated K. pneumoniae-infected mice. These results suggest that SBAs may be an ideal therapeutic target for reducing inflammatory levels in liver abscess and bacteremia by suppressing the NF- $\kappa \mathrm{B}$ pathway.

However, the mechanism by which SBAs repress bacterial antigen-activated NF- $\kappa B$ signaling pathway in the liver is still unclear. Emerging evidence shows that SBAs can regulate host immune systems via the $G$ protein-coupled receptor for bile acids (TGR5), ${ }^{47}$ which is a promising target in the context of bile acid and microbial interactions. TGR5 is differentially expressed in immune cells such as macrophages and glial cells. Studies have shown that TGR5 activation on the macrophage cell membrane inhibits the expression of LPS-induced cytokines. ${ }^{48}$ Sinha et $\mathrm{al}^{25}$ found that in three different colitis models, SBA supplementation alleviated intestinal inflammation, and in-depth exploration revealed that this effect was mediated by TGR5. LCA and DCA inhibit proinflammatory cytokine production in human peripheral-blood-derived macrophages, which are key mediators of intestinal inflammation in ulcerative colitis, by activating the TGR5 receptor. ${ }^{49}$ In conclusion, there is sufficient preclinical evidence to suggest that TGR5 activation plays a beneficial role in the treatment of inflammatory diseases. This study investigated the effect of SBAs on TGR5 activation in liver. As expected, SBAs not only had an up-regulation effect on liver $T G R 5$ gene expression, but also significantly increased the level of TGR5 protein in SBA-treated liver abscess mice. These results suggest that SBAs inhibit the activation of inflammatory signaling pathways by activating hepatic TGR5 expression.

\section{Conclusion}

In summary, $K$. pneumoniae-induced liver abscess and bacteremia mice exhibited significant alteration of the intestinal flora. The change in the community structure of the gut microbiota, such as the decrease in the abundance of Ruminococcaceae, may be the cause of the reduced levels of SBAs. Supplementation with SBAs was shown to improve the general condition of mice with liver abscesses, including survival, liver pathology, and inflammation levels. We also found that oral gavage of SBAs lowered inflammatory levels by reducing the expression of key proteins in the NF- $\mathrm{BB}$ inflammatory signaling pathway. Finally, high TGR5 expression may mediate the role of SBAs. In future work, we aim to use gene editing technology to knock out the TGR5 gene in order to verify whether SBAs exert a beneficial effect through TGR5. Furthermore, we will attempt to analyze the intestinal flora and metabolites in individuals with liver abscesses in order to identify molecular markers for clinical diagnosis of the disease. We believe that the findings of this study will enable the development of novel methods for the treatment of liver abscess and bacteremia.

\section{Abbreviations}

KLA, Klebsiella pneumoniae liver abscess; hvKp, hypervirulent Klebsiella pneumoniae; cKp, classic Klebsiella pneumoniae; SBAs, secondary bile acids; PBAs, primary bile acids; DCA, deoxycholic acid; LCA, lithocholic acid; $\mathrm{NF}-\kappa \mathrm{B}$, nuclear factor- $\kappa \mathrm{B}$; PBS, sterile phosphate-buffered saline; LC-MS, liquid chromatograph-mass spectrometer; H\&E, hematoxylin and eosin; IHC, immunohistochemistry; ANOVA, one-way analysis of variance; PLS-DA, partial least squares-discriminant analysis; TNF, tumor necrosis factor; $\mathrm{MCP}$, monocyte chemotactic protein; $\mathrm{KC}$, keratinocyte-derived chemokine; IP, interferon-induced protein; TGR5, G protein-coupled receptor for bile acids.

\section{Acknowledgment}

The authors are extremely thankful to Yuanyuan Dai and Jiajia Li for their technical support.

\section{Funding}

This work was supported by research grants from the National Natural Science Foundation of China (No81973983), the National Science and Technology Major Project (No. 2017ZX10204401) and the 
Borrowing and Transferring Subsidy Project in 2019, Hefei (J2019Y04).

\section{Disclosure}

The authors report no conflicts of interest in this work.

\section{References}

1. Lin YT, Liu CJ, Fung CP, Tzeng CH. Nosocomial Klebsiella pneumoniae bacteraemia in adult cancer patients-characteristics of neutropenic and non-neutropenic patients. Scand J Infect Dis. 2011;43 (8):603-608. doi:10.3109/00365548.2011.577800

2. Lin YT, Chen TL, Siu LK, Hsu SF, Fung CP. Clinical and microbiological characteristics of community-acquired thoracic empyema or complicated parapneumonic effusion caused by Klebsiella pneumoniae in Taiwan. Eur J Clin Microbiol Infect Dis. 2010;29 (8):1003-1010. doi:10.1007/s10096-010-0961-8

3. Zhang Y, Zhao C, Wang Q, et al. High prevalence of hypervirulent klebsiella pneumoniae infection in China: geographic distribution, clinical characteristics, and antimicrobial resistance. Antimicrob Agents Chemother. 2016;60(10):6115-6120. doi:10.1128/aac.0112716

4. Tsai FC, Huang YT, Chang LY, Wang JT. Pyogenic liver abscess as endemic disease, Taiwan. Emerg Infect Dis. 2008;14(10):1592-1600. doi:10.3201/eid1410.071254

5. Shon AS, Russo TA. Hypervirulent Klebsiella pneumoniae: the next superbug? Future Microbiol. 2012;7(6):669-671. doi:10.2217/ fmb. 12.43

6. Lin YT, Siu LK, Lin JC, et al. Seroepidemiology of Klebsiella pneumoniae colonizing the intestinal tract of healthy Chinese and overseas Chinese adults in Asian countries. BMC Microbiol. 2012;12 (1):13. doi:10.1186/1471-2180-12-13

7. Yeh KM, Kurup A, Siu LK, et al. Capsular serotype K1 or K2, rather than magA and rmpA, is a major virulence determinant for Klebsiella pneumoniae liver abscess in Singapore and Taiwan. J Clin Microbiol. 2007;45(2):466-471. doi:10.1128/jcm.01150-06

8. Chung DR, Lee SS, Lee HR, et al. Emerging invasive liver abscess caused by $\mathrm{K} 1$ serotype Klebsiella pneumoniae in Korea. J Infect. 2007;54(6):578-583. doi:10.1016/j.jinf.2006.11.008

9. Lee CR, Lee JH, Park KS, et al. Antimicrobial resistance of hypervirulent Klebsiella pneumoniae: epidemiology, hypervirulence-associated determinants, and resistance mechanisms. Front Cell Infect Microbiol. 2017;7:483. doi:10.3389/ fcimb.2017.00483

10. Conlon MA, Bird AR. The impact of diet and lifestyle on gut microbiota and human health. Nutrients. 2014;7(1):17-44. doi: $10.3390 /$ nu7010017

11. Duan Y, Llorente C, Lang S, et al. Bacteriophage targeting of gut bacterium attenuates alcoholic liver disease. Nature. 2019;575 (7783):505-511. doi:10.1038/s41586-019-1742-x

12. Mouries J, Brescia P, Silvestri A, et al. Microbiota-driven gut vascular barrier disruption is a prerequisite for non-alcoholic steatohepatitis development. $J$ Hepatol. 2019;71(6):1216-1228. doi:10.1016/j. jhep.2019.08.005

13. Qin N, Yang F, Li A, et al. Alterations of the human gut microbiome in liver cirrhosis. Nature. 2014;513(7516):59-64. doi:10.1038/ nature 13568

14. Bajaj JS, Heuman DM, Hylemon PB, et al. Altered profile of human gut microbiome is associated with cirrhosis and its complications. J Hepatol. 2014;60(5):940-947. doi:10.1016/j.jhep.2013.12.019

15. Holmes E, Li JV, Marchesi JR, Nicholson JK. Gut microbiota composition and activity in relation to host metabolic phenotype and disease risk. Cell Metab. 2012;16(5):559-564. doi:10.1016/j. cmet.2012.10.007
16. de Aguiar Vallim TQ, Tarling EJ, Edwards PA. Pleiotropic roles of bile acids in metabolism. Cell Metab. 2013;17(5):657-669. doi:10.1016/j.cmet.2013.03.013

17. Devlin AS, Fischbach MA. A biosynthetic pathway for a prominent class of microbiota-derived bile acids. Nat Chem Biol. 2015;11 (9):685-690. doi:10.1038/nchembio.1864

18. Stellwag EJ, Hylemon PB. 7Alpha-dehydroxylation of cholic acid and chenodeoxycholic acid by Clostridium leptum. J Lipid Res. 1979;20(3):325-333. doi:10.1016/S0022-2275(20)40615-7

19. Stellwag EJ, Hylemon PB. Characterization of 7-alphadehydroxylase in Clostridium leptum. Am J Clin Nutr. 1978;31 (10Suppl):S243-s247. doi:10.1093/ajcn/31.10.S243

20. Stadler J, Stern HS, Yeung KS, et al. Effect of high fat consumption on cell proliferation activity of colorectal mucosa and on soluble faecal bile acids. Gut. 1988;29(10):1326-1331. doi:10.1136/ gut.29.10.1326

21. Gadaleta RM, van Mil SW, Oldenburg B, Siersema PD, Klomp LW, van Erpecum KJ. Bile acids and their nuclear receptor FXR: relevance for hepatobiliary and gastrointestinal disease. Biochim Biophys Acta. 2010;1801(7):683-692. doi:10.1016/j.bbalip.2010.04.006

22. Bernstein H, Bernstein C, Payne CM, Dvorak K. Bile acids as endogenous etiologic agents in gastrointestinal cancer. World J Gastroenterol. 2009;15(27):3329-3340. doi:10.3748/wjg.15.3329

23. Ward JBJ, Lajczak NK, Kelly OB, et al. Ursodeoxycholic acid and lithocholic acid exert anti-inflammatory actions in the colon. $\mathrm{Am}$ J Physiol Gastrointest Liver Physiol. 2017;312(6):G550-g558. doi:10.1152/ajpgi.00256.2016

24. Winkler ES, Shrihari S, Hykes BL Jr. The intestinal microbiome restricts alphavirus infection and dissemination through a bile acid-type I IFN signaling axis. Cell. 2020;182(4):901-918.e918. doi:10.1016/j.cell.2020.06.029

25. Sinha SR, Haileselassie Y, Nguyen LP, et al. Dysbiosis-induced secondary bile acid deficiency promotes intestinal inflammation. Cell Host Microbe. 2020;27(4):659-670.e655. doi:10.1016/j.chom.2020.01.021

26. Lenz AM, Fairweather M, Peyton JC, Gardner SA, Cheadle WG. Liver injury and abscess formation in secondary murine peritonitis. Inflamm Res. 2011;60(4):337-345. doi:10.1007/s00011-010-0273-6

27. La Frano MR, Hernandez-Carretero A, Weber N, et al. Diet-induced obesity and weight loss alter bile acid concentrations and bile acid-sensitive gene expression in insulin target tissues of C57BL/6J mice. Nutr Res. 2017;46:11-21. doi:10.1016/j.nutres.2017.07.006

28. Wu T, Xu F, Su C, et al. Alterations in the gut microbiome and cecal metabolome during klebsiella pneumoniae-induced pneumosepsis. Front Immunol. 2020;11:1331. doi:10.3389/fimmu.2020.01331

29. Rooks MG, Garrett WS. Gut microbiota, metabolites and host immunity. Nat Rev Immunol. 2016;16(6):341-352. doi:10.1038/ nri.2016.42

30. Ling Z, Liu X, Jia X. Impacts of infection with different toxigenic Clostridium difficile strains on faecal microbiota in children. Sci Rep. 2014;4(1):7485. doi:10.1038/srep07485

31. Ling Z, Liu X, Cheng Y. Decreased diversity of the oral microbiota of patients with hepatitis $\mathrm{b}$ virus-induced chronic liver disease: a pilot project. Sci Rep. 2015;5(1):17098. doi:10.1038/srep17098

32. Lin YT, Tseng KY, Yeh YC, Yang FC, Fung CP, Chen NJ. TREM-1 promotes survival during Klebsiella pneumoniae liver abscess in mice. Infect Immun. 2014;82(3):1335-1342. doi:10.1128/iai.01347-13

33. Lin YT, Liu CJ, Yeh YC, Chen TJ, Fung CP. Ampicillin and amoxicillin use and the risk of Klebsiella pneumoniae liver abscess in Taiwan. J Infect Dis. 2013;208(2):211-217. doi:10.1093/infdis/jit157

34. Fei J, Fu L, Hu B, et al. Obeticholic acid alleviate lipopolys accharide-induced acute lung injury via its anti-inflammatory effects in mice. Int Immunopharmacol. 2019;66:177-184. doi:10.1016/j. intimp.2018.11.005

35. Peermohamed S, Kogilwaimath S. Hypervirulent (hypermucoviscous) Klebsiella pneumoniae causing pyogenic liver abscess. Cmaj. 2018;190(14):E441. doi:10.1503/cmaj.171344 
36. Li J, Jia H, Cai X, et al. An integrated catalog of reference genes in the human gut microbiome. Nat Biotechnol. 2014;32(8):834-841. doi: $10.1038 /$ nbt. 2942

37. Huttenhower C, Gevers D, Knight R, et al.. Structure, function and diversity of the healthy human microbiome. Nature. 2012;486 (7402):207-214. doi:10.1038/nature11234

38. Chen ML, Takeda K, Sundrud MS. Emerging roles of bile acids in mucosal immunity and inflammation. Mucosal Immunol. 2019;12 (4):851-861. doi:10.1038/s41385-019-0162-4

39. Biesalski HK. Nutrition meets the microbiome: micronutrients and the microbiota. Ann N Y Acad Sci. 2016;1372(1):53-64. doi:10.1111/ nyas. 13145

40. Oliphant K, Allen-Vercoe E. Macronutrient metabolism by the human gut microbiome: major fermentation by-products and their impact on host health. Microbiome. 2019;7(1):91. doi:10.1186/s40168-0190704-8

41. Wahlström A, Sayin SI, Marschall HU, Bäckhed F. Intestinal crosstalk between bile acids and microbiota and its impact on host metabolism. Cell Metab. 2016;24(1):41-50. doi:10.1016/j. cmet.2016.05.005

42. Fiorucci S, Biagioli M, Zampella A, Distrutti E. Bile acids activated receptors regulate innate immunity. Front Immunol. 2018;9:1853. doi: $10.3389 /$ fimmu. 2018.01853

43. Inagaki T, Moschetta A, Lee YK, et al. Regulation of antibacterial defense in the small intestine by the nuclear bile acid receptor. Proc Natl Acad Sci U S A. 2006;103(10):3920-3925. doi:10.1073/pnas.0509592103
44. Hoh CH, Tan YH, Gan YH, Torres VJ. Protective role of kupffer cells and macrophages in Klebsiella pneumoniae -induced liver abscess disease. Infect Immun. 2019;87(9). doi:10.1128/iai.00369-19

45. Pikarsky E, Porat RM, Stein I, et al. NF-kappaB functions as a tumour promoter in inflammation-associated cancer. Nature. 2004;431(7007):461-466. doi:10.1038/nature02924

46. D'Acquisto F, Ianaro A. From willow bark to peptides: the ever widening spectrum of NF-kappaB inhibitors. Curr Opin Pharmacol. 2006;6(4):387-392. doi:10.1016/j.coph.2006.02.009

47. Cipriani S, Mencarelli A, Chini MG, et al. The bile acid receptor GPBAR-1 (TGR5) modulates integrity of intestinal barrier and immune response to experimental colitis. PLoS One. 2011;6(10): e25637. doi:10.1371/journal.pone.0025637

48. Keitel V, Donner M, Winandy S, Kubitz R, Häussinger D. Expression and function of the bile acid receptor TGR5 in Kupffer cells. Biochem Biophys Res Commun. 2008;372(1):78-84. doi:10.1016/j. bbrc.2008.04.171

49. Voss OH, Murakami Y, Pena MY, et al. Lipopolysaccharide-induced CD300b receptor binding to toll-like receptor 4 alters signaling to drive cytokine responses that enhance septic shock. Immunity. 2016;44(6):1365-1378. doi:10.1016/j.immuni.2016.05.005
Journal of Inflammation Research

\section{Publish your work in this journal}

The Journal of Inflammation Research is an international, peerreviewed open-access journal that welcomes laboratory and clinical findings on the molecular basis, cell biology and pharmacology of inflammation including original research, reviews, symposium reports, hypothesis formation and commentaries on: acute/chronic inflammation; mediators of inflammation; cellular processes; molecular

\section{Dovepress}

mechanisms; pharmacology and novel anti-inflammatory drugs; clinical conditions involving inflammation. The manuscript management system is completely online and includes a very quick and fair peerreview system. Visit http://www.dovepress.com/testimonials.php to read real quotes from published authors. 